\title{
Learning Dictionaries for Named Entity Recognition using Minimal Supervision
}

\author{
Arvind Neelakantan \\ Department of Computer Science \\ University of Massachusetts, Amherst \\ Amherst, MA, 01003 \\ arvindecs.umass . edu
}

\author{
Michael Collins \\ Department of Computer Science \\ Columbia University \\ New-York, NY 10027, USA \\ mcollinsecs. columbia.edu
}

\begin{abstract}
This paper describes an approach for automatic construction of dictionaries for Named Entity Recognition (NER) using large amounts of unlabeled data and a few seed examples. We use Canonical Correlation Analysis (CCA) to obtain lower dimensional embeddings (representations) for candidate phrases and classify these phrases using a small number of labeled examples. Our method achieves $16.5 \%$ and $11.3 \%$ F-1 score improvement over co-training on disease and virus NER respectively. We also show that by adding candidate phrase embeddings as features in a sequence tagger gives better performance compared to using word embeddings.
\end{abstract}

\section{Introduction}

Several works (e.g., Ratinov and Roth, 2009; Cohen and Sarawagi, 2004) have shown that injecting dictionary matches as features in a sequence tagger results in significant gains in NER performance. However, building these dictionaries requires a huge amount of human effort and it is often difficult to get good coverage for many named entity types. The problem is more severe when we consider named entity types such as gene, virus and disease, because of the large (and growing) number of names in use, the fact that the names are heavily abbreviated and multiple names are used to refer to the same entity (Leaman et al., 2010, Dogan and Lu, 2012). Also, these dictionaries can only be built by domain experts, making the process very expensive.

This paper describes an approach for automatic construction of dictionaries for NER using large amounts of unlabeled data and a small number of seed examples. Our approach consists of two steps. First, we collect a high recall, low precision list of candidate phrases from the large unlabeled data collection for every named entity type using simple rules. In the second step, we construct an accurate dictionary of named entities by removing the noisy candidates from the list obtained in the first step. This is done by learning a classifier using the lower dimensional, real-valued CCA (Hotelling, 1935) embeddings of the candidate phrases as features and training it using a small number of labeled examples. The classifier we use is a binary SVM which predicts whether a candidate phrase is a named entity or not.

We compare our method to a widely used semisupervised algorithm based on co-training (Blum and Mitchell, 1998). The dictionaries are first evaluated on virus (GENIA, 2003) and disease (Dogan and Lu, 2012) NER by using them directly in dictionary based taggers. We also give results comparing the dictionaries produced by the two semi-supervised approaches with dictionaries that are compiled manually. The effectiveness of the dictionaries are also measured by injecting dictionary matches as features in a Conditional Random Field (CRF) based tagger. The results indicate that our approach with minimal supervision produces dictionaries that are comparable to dictionaries compiled manually. Finally, we also compare the quality of the candidate phrase embeddings with word embeddings (Dhillon et al., 2011) by adding them as features in a CRF based sequence tagger.

\section{Background}

We first give background on Canonical Correlation Analysis (CCA), and then give background on 
CRFs for the NER problem.

\subsection{Canonical Correlation Analysis (CCA)}

The input to CCA consists of $n$ paired observations $\left(x_{1}, z_{1}\right), \ldots,\left(x_{n}, z_{n}\right)$ where $x_{i} \in \mathbb{R}^{d_{1}}, z_{i} \in$ $\mathbb{R}^{d_{2}}(\forall i \in\{1,2, \ldots, n\})$ are the feature representations for the two views of a data point. CCA simultaneously learns projection matrices $\Phi_{1} \in$ $\mathbb{R}^{d_{1} \times k}, \Phi_{2} \in \mathbb{R}^{d_{2} \times k}$ ( $k$ is a small number) which are used to obtain the lower dimensional representations $\left(\bar{x}_{1}, \bar{z}_{1}\right), \ldots,\left(\bar{x}_{n}, \bar{z}_{n}\right)$ where $\bar{x}_{i}=\Phi_{1}^{\top} x_{i} \in$ $\mathbb{R}^{k}, \bar{z}_{i}=\Phi_{2}^{\top} z_{i} \in \mathbb{R}^{k}, \forall i \in\{1,2, \ldots, n\} . \Phi_{1}, \Phi_{2}$ are chosen to maximize the correlation between $\bar{x}_{i}$ and $\bar{z}_{i}, \forall i \in\{1,2, \ldots, n\}$.

Consider the setting where we have a label for the data point along with it's two views and either view is sufficient to make accurate predictions. Kakade and Foster (2007) and Sridharan and Kakade (2008) give strong theoretical guarantees when the lower dimensional embeddings from CCA are used for predicting the label of the data point. This setting is similar to the one considered in co-training (Collins and Singer, 1999) but there is no assumption of independence between the two views of the data point. Also, it is an exact algorithm unlike the algorithm given in Collins and Singer (1999). Since we are using lower dimensional embeddings of the data point for prediction, we can learn a predictor with fewer labeled examples.

\subsection{CRFs for Named Entity Recognition}

CRF based sequence taggers have been used for a number of NER tasks (e.g., McCallum and Li, 2003) and in particular for biomedical NER (e.g., McDonald and Pereira, 2005; Burr Settles, 2004) because they allow a great deal of flexibility in the features which can be included. The input to a CRF tagger is a sentence $\left(w_{1}, w_{2}, \ldots, w_{n}\right)$ where $w_{i}, \forall i \in\{1,2, \ldots, n\}$ are words in the sentence. The output is a sequence of tags $y_{1}, y_{2}, \ldots, y_{n}$ where $y_{i} \in\{\mathrm{B}, \mathrm{I}, \quad \mathrm{O}\}, \forall i \in\{1,2, \ldots, n\} . \mathrm{B}$ is the tag given to the first word in a named entity, I is the tag given to all words except the first word in a named entity and $O$ is the tag given to all other words. We used the standard NER baseline features (e.g., Dhillon et al., 2011; Ratinov and Roth, 2009) which include:

- Current Word $w_{i}$ and its lexical features which include whether the word is capitalized and whether all the characters are cap- italized. Prefix and suffixes of the word $w_{i}$ were also added.

- Word tokens in window of size two around the current word which include $w_{i-2}, w_{i-1}, w_{i+1}, w_{i+2}$ and also the capitalization pattern in the window.

- Previous two predictions $y_{i-1}$ and $y_{i-2}$.

The effectiveness of the dictionaries are evaluated by adding dictionary matches as features along with the baseline features (Ratinov and Roth, 2009: Cohen and Sarawagi, 2004) in the CRF tagger. We also compared the quality of the candidate phrase embeddings with the word-level embeddings by adding them as features (Dhillon et al., 2011) along with the baseline features in the CRF tagger.

\section{Method}

This section describes the two steps in our approach: obtaining candidate phrases and classifying them.

\subsection{Obtaining Candidate Phrases}

We used the full text of 110,369 biomedical publications in the BioMed Central corpus 1 to get the high recall, low precision list of candidate phrases. The advantages of using this huge collection of publications are obvious: almost all (including rare) named entities related to the biomedical domain will be mentioned and contains more recent developments than a structured resource like Wikipedia. The challenge however is that these publications are unstructured and hence it is a difficult task to construct accurate dictionaries using them with minimal supervision.

The list of virus candidate phrases were obtained by extracting phrases that occur between "the" and "virus" in the simple pattern "the ... virus" during a single pass over the unlabeled document collection. This noisy list had a lot of virus names such as influenza, human immunodeficiency and Epstein-Barr along with phrases that are not virus names, like mutant, same, new, and so on.

A similar rule like "the ... disease" did not give a good coverage of disease names since it is not the common way of how diseases are mentioned in publications. So we took a different approach

\footnotetext{
${ }^{1}$ The corpus can be downloaded at http://www.biomedcentral.com/about/datamining
} 
to obtain the noisy list of disease names. We collected every sentence in the unlabeled data collection that has the word "disease" in it and extracted noun phrases ${ }^{2}$ following the patterns "diseases like ....", "diseases such as ...." , "diseases including ...." , "diagnosed with ....", "patients with ...." and "suffering from ....".

\subsection{Classification of Candidate Phrases}

Having found the list of candidate phrases, we now describe how noisy words are filtered out from them. We gather (spelling, context) pairs for every instance of a candidate phrase in the unlabeled data collection. spelling refers to the candidate phrase itself while context includes three words each to the left and the right of the candidate phrase in the sentence. The spelling and the context of the candidate phrase provide a natural split into two views which multi-view algorithms like co-training and CCA can exploit. The only supervision in our method is to provide a few spelling seed examples (10 in the case of virus, 18 in the case of disease), for example, human immunodeficiency is a virus and mutant is not a virus.

\subsubsection{Approach using CCA embeddings}

We use CCA described in the previous section to obtain lower dimensional embeddings for the candidate phrases using the (spelling, context) views. Unlike previous works such as Dhillon et al. (2011) and Dhillon et al. (2012), we use CCA to learn embeddings for candidate phrases instead of all words in the vocabulary so that we don't miss named entities which have two or more words.

Let the number of (spelling, context) pairs be $n$ (sum of total number of instances of every candidate phrase in the unlabeled data collection). First, we map the spelling and context to highdimensional feature vectors. For the spelling view, we define a feature for every candidate phrase and also a boolean feature which indicates whether the phrase is capitalized or not. For the context view, we use features similar to Dhillon et al. (2011) where a feature for every word in the context in conjunction with its position is defined. Each of the $n$ (spelling, context) pairs are mapped to a pair of high-dimensional feature vectors to get $n$ paired observations $\left(x_{1}, z_{1}\right), \ldots,\left(x_{n}, z_{n}\right)$ with $x_{i} \in \mathbb{R}^{d_{1}}, z_{i} \in \mathbb{R}^{d_{2}}, \forall i \in\{1,2, \ldots, n\}\left(d_{1}, d_{2}\right.$ are the feature space dimensions of the spelling

\footnotetext{
${ }^{2}$ Noun phrases were obtained using http://www.umiacs.umd.edu/ hal/TagChunk/
}

and context view respectively). Using $\mathrm{CCA}^{3}$, we learn the projection matrices $\Phi_{1} \in \mathbb{R}^{d_{1} \times k}, \Phi_{2} \in$ $\mathbb{R}^{d_{2} \times k}\left(k<<d_{1}\right.$ and $\left.k<<d_{2}\right)$ and obtain spelling view projections $\bar{x}_{i}=\Phi_{1}^{\top} x_{i} \in \mathbb{R}^{k}, \forall i \in$ $\{1,2, \ldots, n\}$. The k-dimensional spelling view projection of any instance of a candidate phrase is used as it's embedding 4

The k-dimensional candidate phrase embeddings are used as features to learn a binary SVM with the seed spelling examples given in figure 1 as training data. The binary SVM predicts whether a candidate phrase is a named entity or not. Since the value of $\mathrm{k}$ is small, a small number of labeled examples are sufficient to train an accurate classifier. The learned SVM is used to filter out the noisy phrases from the list of candidate phrases obtained in the previous step.

To summarize, our approach for classifying candidate phrases has the following steps:

- Input: $n$ (spelling, context) pairs, spelling seed examples.

- Each of the $n$ (spelling, context) pairs are mapped to a pair of high-dimensional feature vectors to get $n$ paired observations $\left(x_{1}, z_{1}\right), \ldots,\left(x_{n}, z_{n}\right)$ with $x_{i} \in \mathbb{R}^{d_{1}}, z_{i} \in$ $\mathbb{R}^{d_{2}}, \forall i \in\{1,2, \ldots, n\}$.

- Using CCA, we learn the projection matrices $\Phi_{1} \in \mathbb{R}^{d_{1} \times k}, \Phi_{2} \in \mathbb{R}^{d_{2} \times k}$ and obtain spelling view projections $\bar{x}_{i}=\Phi_{1}^{\top} x_{i} \in$ $\mathbb{R}^{k}, \forall i \in\{1,2, \ldots, n\}$.

- The embedding of a candidate phrase is given by the k-dimensional spelling view projection of any instance of the candidate phrase.

- We learn a binary SVM with the candidate phrase embeddings as features and the spelling seed examples given in figure 1 as training data. Using this SVM, we predict whether a candidate phrase is a named entity or not.

\subsubsection{Approach based on Co-training}

We discuss here briefly the DL-CoTrain algorithm (Collins and Singer, 1999) which is based on cotraining (Blum and Mitchell, 1998), to classify

${ }^{3}$ Similar to Dhillon et al. (2012) we used the method given in Halko et al. (2011) to perform the SVD computation in CCA for practical considerations.

${ }^{4}$ Note that a candidate phrase gets the same spelling view projection across it's different instances since the spelling features of a candidate phrase are identical across it's instances. 
- Virus seed spelling examples

- Virus Names: human immunodeficiency, hepatitis C, influenza, Epstein-Barr, hepatitis B

- Non-virus Names: mutant, same, wild type, parental, recombinant

- Disease seed spelling examples

- Disease Names: tumor, malaria, breast cancer, cancer, IDDM, DM, A-T, tumors, VHL

- Non-disease Names: cells, patients, study, data, expression, breast, BRCA1, protein, mutant

Figure 1: Seed spelling examples

candidate phrases. We compare our approach using CCA embeddings with this approach. Here, two decision list of rules are learned simultaneously one using the spelling view and the other using the context view. The rules using the spelling view are of the form: full-string=human immunodeficiency $\rightarrow$ Virus, full-string $=$ mutant $\rightarrow$ Not_a_virus and so on. In the context view, we used bigram 5 rules where we considered all possible bigrams using the context. The rules are of two types: one which gives a positive label, for example, full-string=human immunodeficiency $\rightarrow$ Virus and the other which gives a negative label, for example, full-string=mutant $\rightarrow$ Not_a_virus. The DL-CoTrain algorithm is as follows:

- Input: (spelling, context) pairs for every instance of a candidate phrase in the corpus, $m$ specifying the number of rules to be added in every iteration, precision threshold $\epsilon$, spelling seed examples.

- Algorithm:

1. Initialize the spelling decision list using the spelling seed examples given in figure 1 and set $i=1$.

2. Label the entire input collection using the learned decision list of spelling rules.

3. Add $i \times m$ new context rules of each type to the decision list of context rules using the current labeled data. The rules are added using the same criterion as given in Collins and Singer (1999), i.e., among the rules whose strength is greater than the precision threshold $\epsilon$, the ones which are seen more often with the corresponding label in the input data collection are added.

\footnotetext{
${ }^{5}$ We tried using unigram rules but they were very weak predictors and the performance of the algorithm was poor when they were considered.
}

4. Label the entire input collection using the learned decision list of context rules.

5. Add $i \times m$ new spelling rules of each type to the decision list of spelling rules using the current labeled data. The rules are added using the same criterion as in step 3 . Set $i=i+1$. If rules were added in the previous iteration, return to step 2 .

The algorithm is run until no new rules are left to be added. The spelling decision list along with its strength (Collins and Singer, 1999) is used to construct the dictionaries. The phrases present in the spelling rules which give a positive label and whose strength is greater than the precision threshold, were added to the dictionary of named entities. We found the parameters $\mathrm{m}$ and $\epsilon$ difficult to tune and they could significantly affect the performance of the algorithm. We give more details regarding this in the experiments section.

\section{Related Work}

Previously, Collins and Singer (1999) introduced a multi-view, semi-supervised algorithm based on co-training (Blum and Mitchell, 1998) for collecting names of people, organizations and locations. This algorithm makes a strong independence assumption about the data and employs many heuristics to greedily optimize an objective function. This greedy approach also introduces new parameters that are often difficult to tune.

In other works such as Toral and Muñoz (2006) and Kazama and Torisawa (2007) external structured resources like Wikipedia have been used to construct dictionaries. Even though these methods are fairly successful they suffer from a number of drawbacks especially in the biomedical domain. The main drawback of these approaches is that it is very difficult to accurately disambiguate ambiguous entities especially when the entities are 
abbreviations (Kazama and Torisawa, 2007). For example, $D M$ is the abbreviation for the disease Diabetes Mellitus and the disambiguation page for $D M$ in Wikipedia associates it to more than 50 categories since $D M$ can be expanded to Doctor of Management, Dichroic mirror, and so on, each of it belonging to a different category. Due to the rapid growth of Wikipedia, the number of entities that have disambiguation pages is growing fast and it is increasingly difficult to retrieve the article we want. Also, it is tough to understand these approaches from a theoretical standpoint.

Dhillon et al. (2011) used CCA to learn word embeddings and added them as features in a sequence tagger. They show that CCA learns better word embeddings than $\mathrm{CW}$ embeddings (Collobert and Weston , 2008), Hierarchical log-linear (HLBL) embeddings (Mnih and Hinton, 2007) and embeddings learned from many other techniques for NER and chunking. Unlike PCA, a widely used dimensionality reduction technique, CCA is invariant to linear transformations of the data. Our approach is motivated by the theoretical result in Kakade and Foster (2007) which is developed in the co-training setting. We directly use the CCA embeddings to predict the label of a data point instead of using them as features in a sequence tagger. Also, we learn CCA embeddings for candidate phrases instead of all words in the vocabulary since named entities often contain more than one word. Dhillon et al. (2012) learn a multi-class SVM using the CCA word embeddings to predict the POS tag of a word type. We extend this technique to NER by learning a binary SVM using the CCA embeddings of a high recall, low precision list of candidate phrases to predict whether a candidate phrase is a named entity or not.

\section{Experiments}

In this section, we give experimental results on virus and disease NER.

\subsection{Data}

The noisy lists of both virus and disease names were obtained from the BioMed Central corpus. This corpus was also used to get the collection of (spelling, context) pairs which are the input to the CCA procedure and the DL-CoTrain algorithm described in the previous section. We obtained CCA embeddings for the 100,000 most frequently oc- curring word types in this collection along with every word type present in the training and development data of the virus and the disease NER dataset. These word embeddings are similar to the ones described in Dhillon et al. (2011) and Dhillon et al. (2012).

We used the virus annotations in the GENIA corpus (GENIA, 2003) for our experiments. The dataset contains 18,546 annotated sentences. We randomly selected 8,546 sentences for training and the remaining sentences were randomly split equally into development and testing sentences. The training sentences are used only for experiments with the sequence taggers. Previously, Zhang et al. (2004) tested their HMM-based named entity recognizer on this data. For disease NER, we used the recent disease corpus (Dogan and Lu, 2012) and used the same training, development and test data split given by them. We used a sentence segmenter ${ }^{6}$ to get sentence segmented data and Stanford Tokenizer 7 to tokenize the data. Similar to Dogan and Lu (2012), all the different disease categories were flattened into one single category of disease mentions. The development data was used to tune the hyperparameters and the methods were evaluated on the test data.

\subsection{Results using a dictionary-based tagger}

First, we compare the dictionaries compiled using different methods by using them directly in a dictionary-based tagger. This is a simple and informative way to understand the quality of the dictionaries before using them in a CRF-tagger. Since these taggers can be trained using a handful of training examples, we can use them to build NER systems even when there are no labeled sentences to train. The input to a dictionary tagger is a list of named entities and a sentence. If there is an exact match between a phrase in the input list to the words in the given sentence then it is tagged as a named entity. All other words are labeled as non-entities. We evaluated the performance of the following methods for building dictionaries:

- Candidate List: This dictionary contains all the candidate phrases that were obtained using the method described in Section 3.1. The noisy list of virus candidates and disease candidates had 3,100 and 60,080 entries respectively.

\footnotetext{
${ }^{6}$ https://pypi.python.org/pypi/text-sentence/0.13

${ }^{7} \mathrm{http}: / /$ nlp.stanford.edu/software/tokenizer.shtml
} 


\begin{tabular}{|c|c|c|c|c|c|c|}
\hline \multirow{2}{*}{ Method } & \multicolumn{3}{|c|}{ Virus NER } & \multicolumn{3}{c|}{ Disease NER } \\
\cline { 2 - 7 } & Precision & Recall & F-1 Score & Precision & Recall & F-1 Score \\
\hline Candidate List & 2.20 & $\mathbf{6 9 . 5 8}$ & 4.27 & 4.86 & $\mathbf{6 0 . 3 2}$ & 8.99 \\
\hline Manual & 42.69 & 68.75 & 52.67 & 51.39 & 45.08 & $\mathbf{4 8 . 0 3}$ \\
\hline Co-Training & 48.33 & 66.46 & 55.96 & $\mathbf{5 8 . 8 7}$ & 23.17 & 33.26 \\
\hline CCA & $\mathbf{5 7 . 2 4}$ & 68.33 & $\mathbf{6 2 . 3 0}$ & 38.34 & 44.55 & 41.21 \\
\hline
\end{tabular}

Table 1: Precision, recall, F- 1 scores of dictionary-based taggers

- Manual: Manually constructed dictionaries, which requires a large amount of human effort, are employed for the task. We used the list of virus names given in Wikipedie ${ }^{8}$. Unfortunately, abbreviations of virus names are not present in this list and we could not find any other more complete list of virus names. Hence, we constructed abbreviations by concatenating the first letters of all the strings in a virus name, for every virus name given in the Wikipedia list.

For diseases, we used the list of disease names given in the Unified Medical Language System (UMLS) Metathesaurus. This dictionary has been widely used in disease NER (e.g., Dogan and Lu, 2012; Leaman et al., 2010)

- Co-Training: The dictionaries are constructed using the DL-CoTrain algorithm described previously. The parameters used were $m=5$ and $\epsilon=0.95$ as given in Collins and Singer (1999). The phrases present in the spelling rules which give a positive label and whose strength is greater than the precision threshold, were added to the dictionary of named entities.

In our experiment to construct a dictionary of virus names, the algorithm stopped after just 12 iterations and hence the dictionary had only 390 virus names. This was because there were no spelling rules with strength greater than 0.95 to be added. We tried varying both the parameters but in all cases, the algorithm did not progress after a few iterations. We adopted a simple heuristic to increase the coverage of virus names by using the strength of the spelling rules obtained after the $12^{\text {th }}$ iteration. All spelling rules that give a positive

\footnotetext{
${ }^{8} \mathrm{http} / / /$ en.wikipedia.org/wiki/List_of_viruses

${ }^{9}$ The list of disease names from UMLS can be found at https://sites.google.com/site/fmchowdhury2/bioenex
}

label and which has a strength greater than $\theta$ were added to the decision list of spelling rules. The phrases present in these rules are added to the dictionary. We picked the $\theta$ parameter from the set $[0.1,0.2,0.3,0.4,0.5$, $0.6,0.7,0.8,0.9]$ using the development data.

The co-training algorithm for constructing the dictionary of disease names ran for close to 50 iterations and hence we obtained better coverage for disease names. We still used the same heuristic of adding more named entities using the strength of the rule since it performed better.

- CCA: Using the CCA embeddings of the candidate phrases ${ }^{10}$ as features we learned a binary SVM ${ }^{11}$ to predict whether a candidate phrase is a named entity or not. We considered using 10 to 30 dimensions of candidate phrase embeddings and the regularizer was picked from the set $[0.0001,0.001,0.01,0.1$, $1,10,100]$. Both the regularizer and the number of dimensions to be used were tuned using the development data.

Table 1 gives the results of the dictionary based taggers using the different methods described above. As expected, when the noisy list of candidate phrases are used as dictionaries the recall of the system is quite high but the precision is very low. The low precision of the Wikipedia virus lists was due to the heuristic used to obtain abbreviations which produced a few noisy abbreviations but this heuristic was crucial to get a high recall. The list of disease names from UMLS gives a low recall because the list does not contain many disease abbreviations and composite disease mentions such as breast and ovarian cancer. The pres-

\footnotetext{
${ }^{10}$ The performance of the dictionaries learned from word embeddings was very poor and we do not report it's performance here.

${ }^{11}$ we used LIBSVM (http://www.csie.ntu.edu.tw/ cjlin/libsvm/ in our SVM experiments
} 

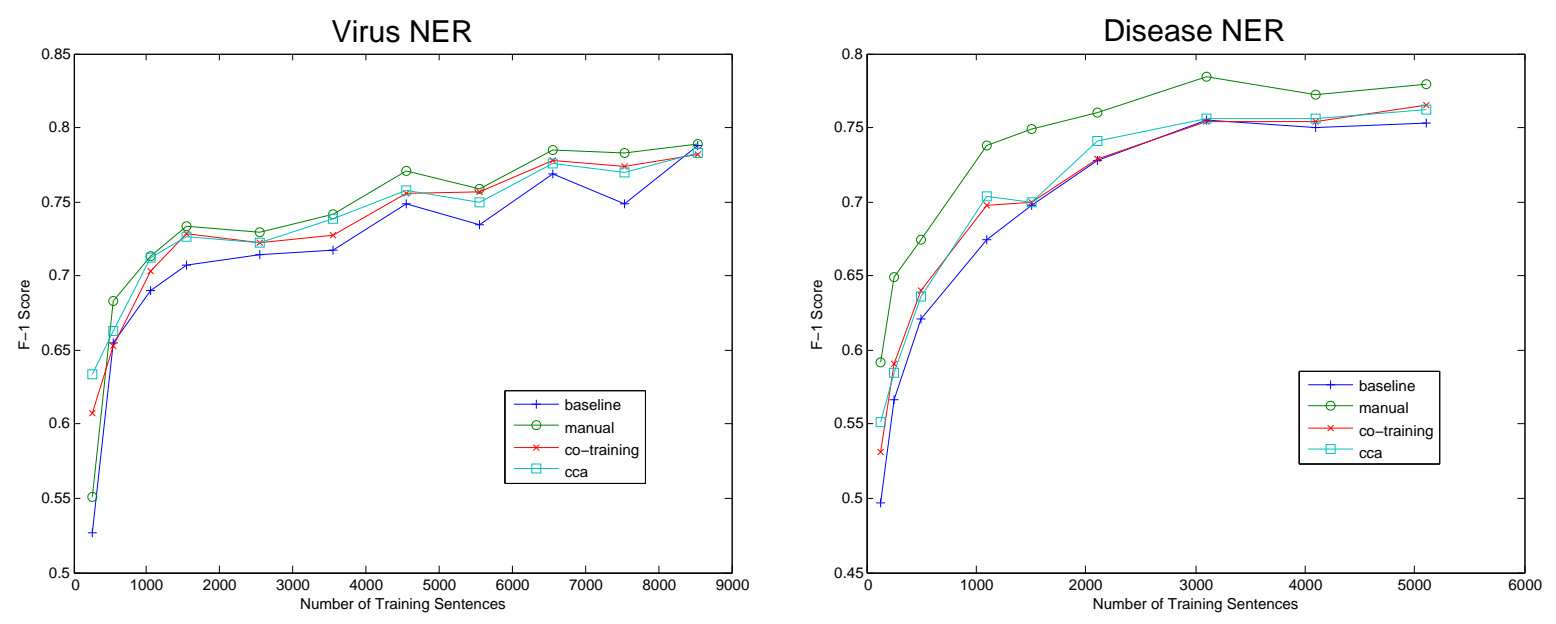

Figure 2: Virus and Disease NER F-1 scores for varying training data size when dictionaries obtained from different methods are injected

ence of ambiguous abbreviations affected the accuracy of this dictionary.

The virus dictionary constructed using the CCA embeddings was very accurate and the false positives were mainly due to ambiguous phrases, for example, in the phrase HIV replication, HIV which usually refers to the name of a virus is tagged as a RNA molecule. The accuracy of the disease dictionary produced using CCA embeddings was mainly affected by noisy abbreviations.

We can see that the dictionaries obtained using CCA embeddings perform better than the dictionaries obtained from co-training on both disease and virus NER even after improving the cotraining algorithm's coverage using the heuristic described in this section. It is important to note that the dictionaries constructed using the CCA embeddings and a small number of labeled examples performs competitively with dictionaries that are entirely built by domain experts. These results show that by using the CCA based approach we can build NER systems that give reasonable performance even for difficult named entity types with almost no supervision.

\subsection{Results using a CRF tagger}

We did two sets of experiments using a CRF tagger. In the first experiment, we add dictionary features to the CRF tagger while in the second experiment we add the embeddings as features to the CRF tagger. The same baseline model is used in both the experiments whose features are described in Section 2.2. For both the $\mathrm{CRF}^{12}$ experiments the regularizers from the set $[0.0001,0.001,0.01$, $0.1,1.0,10.0]$ were considered and it was tuned on the development set.

\subsubsection{Dictionary Features}

Here, we inject dictionary matches as features (e.g., Ratinov and Roth, 2009; Cohen and Sarawagi, 2004) in the CRF tagger. Given a dictionary of named entities, every word in the input sentence has a dictionary feature associated with it. When there is an exact match between a phrase in the dictionary with the words in the input sentence, the dictionary feature of the first word in the named entity is set to B and the dictionary feature of the remaining words in the named entity is set to $I$. The dictionary feature of all the other words in the input sentence which are not part of any named entity in the dictionary is set to $O$. The effectiveness of the dictionaries constructed from various methods are compared by adding dictionary match features to the CRF tagger. These dictionary match features were added along with the baseline features.

Figure 2 indicates that the dictionary features in general are helpful to the CRF model. We can see that the dictionaries produced from our approach using CCA are much more helpful than the dictionaries produced from co-training especially when there are fewer labeled sentences to train. Similar to the dictionary tagger experiments discussed

\footnotetext{
${ }^{12}$ We used CRFsuite (www.chokkan.org/software/crfsuite/) for our experiments with CRFs.
} 

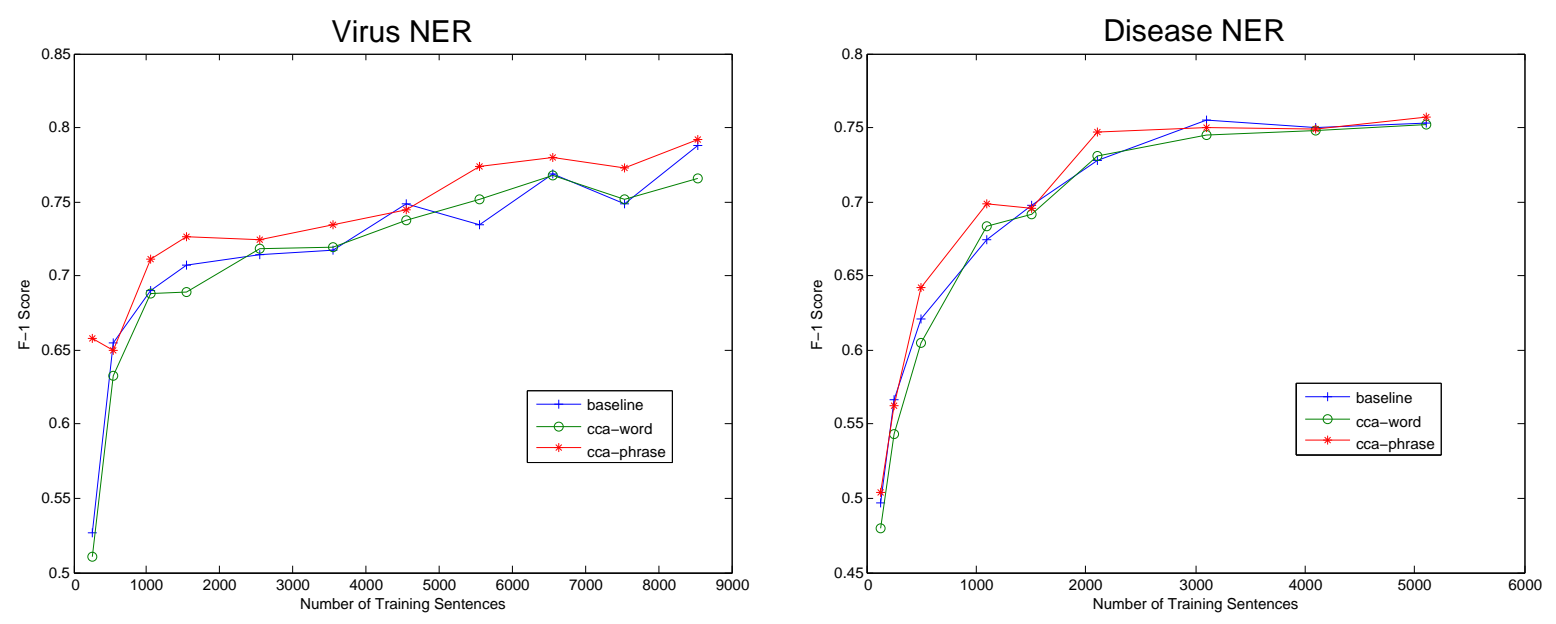

Figure 3: Virus and Disease NER F-1 scores for varying training data size when embeddings obtained from different methods are used as features

previously, the dictionaries produced from our approach performs competitively with dictionaries that are entirely built by domain experts.

\subsubsection{Embedding Features}

The quality of the candidate phrase embeddings are compared with word embeddings by adding the embeddings as features in the CRF tagger. Along with the baseline features, CCA-word model adds word embeddings as features while the CCA-phrase model adds candidate phrase embeddings as features. CCA-word model is similar to the one used in Dhillon et al. (2011).

We considered adding 10, 20, 30, 40 and 50 dimensional word embeddings as features for every training data size and the best performing model on the development data was picked for the experiments on the test data. For candidate phrase embeddings we used the same number of dimensions that was used for training the SVMs to construct the best dictionary.

When candidate phrase embeddings are obtained using CCA, we do not have embeddings for words which are not in the list of candidate phrases. Also, a candidate phrase having more than one word has a joint representation, i.e., the phrase "human immunodeficiency" has a lower dimensional representation while the words "human" and "immunodeficiency" do not have their own lower dimensional representations (assuming they are not part of the candidate list). To overcome this issue, we used a simple technique to differentiate between candidate phrases and the rest of the words. Let $x$ be the highest real valued candidate phrase embedding and the candidate phrase embedding be a $d$ dimensional real valued vector. If a candidate phrase occurs in a sentence, the embeddings of that candidate phrase are added as features to the first word of that candidate phrase. If the candidate phrase has more than one word, the other words in the candidate phrase are given an embedding of dimension $d$ with each dimension having the value $2 \times x$. All the other words are given an embedding of dimension $d$ with each dimension having the value $4 \times x$.

Figure 3 shows that almost always the candidate phrase embeddings help the CRF model. It is also interesting to note that sometimes the wordlevel embeddings have an adverse affect on the performance of the CRF model. The CCA-phrase model performs significantly better than the other two models when there are fewer labeled sentences to train and the separation of the candidate phrases from the other words seems to have helped the CRF model.

\section{Conclusion}

We described an approach for automatic construction of dictionaries for NER using minimal supervision. Compared to the previous approaches, our method is free from overly-stringent assumptions about the data, uses SVD that can be solved exactly and achieves better empirical performance. Our approach which uses a small number of seed examples performs competitively with dictionaries that are compiled manually. 


\section{Acknowledgments}

We are grateful to Alexander Rush, Alexandre Passos and the anonymous reviewers for their useful feedback. This work was supported by the Intelligence Advanced Research Projects Activity (IARPA) via Department of Interior National Business Center (DoI/NBC) contract number D11PC20153. The U.S. Government is authorized to reproduce and distribute reprints for Governmental purposes notwithstanding any copyright annotation thereon. The views and conclusions contained herein are those of the authors and should not be interpreted as necessarily representing the official policies or endorsements, either expressed or implied, of IARPA, DoI/NBC, or the U.S. Government.

\section{References}

[McCallum and Li2003] Andrew McCallum and Wei Li. Early Results for Named Entity Recognition with Conditional Random Fields, Feature Induction and Web-Enhanced Lexicons. 2003. Conference on Natural Language Learning (CoNLL).

[Mnih and Hinton2007] Andriy Mnih and Geoffrey Hinton. Three New Graphical Models for Statistical Language Modelling. 2007. International Conference on Machine learning (ICML).

[Toral and Muñoz2006] Antonio Toral and Rafael Muñoz. A proposal to automatically build and maintain gazetteers for Named Entity Recognition by using Wikipedia. 2006. Workshop On New Text Wikis And Blogs And Other Dynamic Text Sources.

[Blum and Mitchell1998] Avrin Blum and Tom M. Mitchell. Combining Labeled and Unlabeled Data with Co-Training. 1998. Conference on Learning Theory (COLT).

[Burr Settles2004] Burr Settles. Biomedical Named Entity Recognition Using Conditional Random Fields and Rich Feature Sets. 2004. International Joint Workshop on Natural Language Processing in Biomedicine and its Applications (NLPBA).

[Hotelling1935] H. Hotelling. Canonical correlation analysis (cca) 1935. Journal of Educational Psychology.

[Zhang et al.2004] Jie Zhang, Dan Shen, Guodong Zhou, Jian Su and Chew-Lim Tan. Enhancing HMM-based Biomedical Named Entity Recognition by Studying Special Phenomena. 2004. Journal of Biomedical Informatics.

[GENIA2003] Jin-Dong Kim, Tomoko Ohta, Yuka Tateisi and Jun'ichi Tsujii. GENIA corpus - a semantically annotated corpus for bio-textmining. 2003. ISMB.
[Kazama and Torisawa2007] Junichi Kazama and Kentaro Torisawa. Exploiting Wikipedia as External Knowledge for Named Entity Recognition. 2007. Association for Computational Linguistics (ACL).

[Sridharan and Kakade2008] Karthik Sridharan and Sham M. Kakade. An Information Theoretic Framework for Multi-view Learning. 2008. Conference on Learning Theory (COLT).

[Ratinov and Roth2009] Lev Ratinov and Dan Roth. Design Challenges and Misconceptions in Named Entity Recognition. 2009. Conference on Natural Language Learning (CoNLL).

[Collins and Singer1999] Michael Collins and Yoram Singer. Unsupervised Models for Named Entity Classification. 1999. In Proceedings of the Joint SIGDAT Conference on Empirical Methods in Natural Language Processing and Very Large Corpora.

[Halko et al.2011] Nathan Halko, Per-Gunnar Martinsson, Joel A. Tropp. Finding structure with randomness: Probabilistic algorithms for constructing approximate matrix decompositions. 2011. Society for Industrial and Applied Mathematics.

[Dhillon et al.2011] Paramveer S. Dhillon, Dean Foster and Lyle Ungar. Multi-View Learning of Word Embeddings via CCA. 2011. Advances in Neural Information Processing Systems (NIPS).

[Dhillon et al.2012] Paramveer Dhillon, Jordan Rodu, Dean Foster and Lyle Ungar. Two Step CCA: A new spectral method for estimating vector models of words. 2012. International Conference on Machine learning (ICML).

[Dogan and Lu2012] Rezarta Islamaj Dogan and Zhiyong Lu. An improved corpus of disease mentions in PubMed citations. 2012. Workshop on Biomedical Natural Language Processing, Association for Computational Linguistics (ACL).

[Leaman et al.2010] Robert Leaman, Christopher Miller and Graciela Gonzalez. Enabling Recognition of Diseases in Biomedical Text with Machine Learning: Corpus and Benchmark. 2010. Workshop on Biomedical Natural Language Processing, Association for Computational Linguistics (ACL).

[Collobert and Weston 2008] Ronan Collobert and Jason Weston. A unified architecture for natural language processing: deep neural networks with multitask learning. 2008. International Conference on Machine learning (ICML).

[McDonald and Pereira2005] Ryan McDonald and Fernando Pereira. Identifying Gene and Protein Mentions in Text Using Conditional Random Fields. 2005. BMC Bioinformatics.

[Kakade and Foster 2007] Sham M. Kakade and Dean P. Foster. Multi-view regression via canonical correlation analysis. 2007. Conference on Learning Theory (COLT). 
[Cohen and Sarawagi2004] William W. Cohen and Sunita Sarawagi. Exploiting Dictionaries in Named Entity Extraction: Combining Semi-Markov Extraction Processes and Data Integration Methods. 2004. Semi-Markov Extraction Processes and Data Integration Methods, Proceedings of KDD. 